\title{
Metrics for Determining the Frequency Stability Limits of a Power System: A GB Case Study
}

\author{
Marcel Nedd, Waqquas Bukhsh, Callum MacIver, Keith Bell \\ Dept. of Electronic and Electrical Engineering \\ University of Strathclyde \\ Glasgow, United Kingdom
}

\begin{abstract}
The changing power landscape introduces concerns about frequency management in a power system with significant amounts of non-synchronous sources of power. In islanded power systems like Great Britain and Ireland, electricity system operators are sometimes forced to undertake very expensive redispatch actions, including curtailing large amounts of renewable generation to meet statutory frequency stability constraints. Consequently, there is an imminent need to understand and quantify the limits that these constraints pose on the power system and develop metrics that can be easily integrated into current system planning and operational paradigm. This paper proposes three such metrics for quantifying the containment limits of a power system at a given operating point. The paper further argues that while the penetration of non-synchronous dispatch can indeed be used as the basis of a metric to define the containment limits of a power system, it does not account for variations in the contributions of other containment factors such as inertia. To address the aforementioned issue two alternatives are proposed: the first defines the containment limits of a power system without direct reference to penetration of non-synchronous power, instead it determines a relationship in terms of critical inertia. The second alternative improves upon the first and it considers the components of frequency stability constraints, offering an increased degree of flexibility in quantifying containment limits, and understanding the influence that certain key factors have on frequency containment.
\end{abstract}

Index Terms-- Frequency Containment, Frequency Management, Frequency Response, Frequency Stability, Low Inertia, Nonsynchronous Penetration Limits.

\section{INTRODUCTION}

The market and favourable regulatory regime for renewables is driving power systems towards increasing levels of power delivered by non-synchronous technologies. The European Commission is pushing for increased interconnection to optimally utilize the potential of installed renewable generation. They have set an ambitious target of $15 \%$ interconnection import capacity by 2030, relative to the installed capacity in a country [1]. This results in an increasing penetration of power delivered via non-synchronous technologies such as solar photovoltaic (PV) arrays, converterconnected wind power plants, and high voltage direct current (HVDC) interconnectors that presents a greater challenge for islanded power systems like Great Britain (GB) and Ireland, where system operators are sometimes forced to curtail significant amounts of renewable generation to meet statutory stability constraints $[2,3]$. In particular, following a credible loss of infeed or demand event, the rate of change of frequency (RoCoF) and maximum frequency deviation from nominal frequency must still be kept within acceptable bounds. Consequently, there is a need to understand the limits that these constraints impose on the penetration of non-synchronous generation in the power system, at any given time.

At present, non-synchronous generation penetration limits in the GB system are defined in terms of inertia, which is calculated via the swing equation [4]. In Ireland, the nonsynchronous dispatch is managed using the System NonSynchronous Penetration (SNSP) ratio [5]. The Irish system operator, EirGrid, has an operational policy that limits the proportion of demand that can be met at any one time from nonsynchronous sources based on the SNSP, set in 2018 to $65 \%$ [6]. A key limitation of this approach is that it does not consider the variability of inertia in the power system. For instance, a 20 GW scenario with $20 \%$ penetration of non-synchronous dispatch could have different amounts of total system inertia, depending on the inertia contribution of the synchronous machines connected to the power system at the time. The inertia is of great importance since it affects frequency behaviour and whether, or not, a credible loss event is contained within acceptable bounds. Although this can be addressed to a certain degree by applying the swing equation to determine the inertia limit, this expression only considers instantaneous RoCoF, as demonstrated by the authors in [7]. Furthermore, the SNSP does not account for variability in the amount, or type, of frequency response services available at the time of the event, which can change from one operation scenario to another.

This paper highlights the importance of rigorous modelling approaches to determine the penetration limits, as 
underestimating these limits increases the cost of meeting energy demand and overestimating the limits poses a threat to security of supply. Moreover, accurate determination of penetration limits is extremely important in informing the development of new operational policies and ancillary service products, to allow more non-synchronous renewables to be accommodated, reduce curtailment of wind power and imports, and meet objectives such as the GB system operator's commitment to enabling zero carbon operation by 2025 [8].

The main contributions of this paper are as follows:

- $\quad$ a frequency stability model is proposed and validated that includes a representation of the key attributes of the power system that influence frequency behaviour during a power imbalance;

- proposal of a metric that, for a given demand value, provides the limit of inertia in the power system required to meet acceptable frequency conditions and RoCoF limits;

- comparison of the metric with apparent limits of nonsynchronous sources of power required to meet similar acceptable frequency conditions and RoCoF limits;

- $\quad$ proposal of a metric that individually considers RoCoF limits and acceptable frequency conditions, while providing flexibility to understand and quantify the impact of three key factors influencing frequency containment; and

- demonstration of the proposed metrics using the frequency stability model for GB in 2025.

The rest of this paper is structured as follows: Section II discusses frequency management in GB, highlighting current response services, RoCoF limits and acceptable frequency conditions; Section III describes a frequency stability model, validated using a recent event in GB; Section IV presents and discusses three metrics for defining containment limits in the power system; and the paper concludes in Section V.

\section{FREQUENCY MANAGEMENT IN GB}

Power systems with an alternating current (AC) are intended to be operated within given limits for the frequency at which currents alternate. A rise or fall in system frequency is an indication of imbalance between generation and demand on the system that, if not addressed, is likely to lead to frequency instability and ultimately a blackout [9]. Demand for electricity changes continuously and automatic controls are used to modulate power production or consumption from a subset of generators, or large consumers, in order to regulate system frequency; and as the control signal is system frequency, the modulation is called frequency response. In addition to natural variation in demand and generation, power systems are subject to unplanned disturbances such as fault outages of generators, interconnectors to neighbouring countries, or large loads. A common convention is that power systems are operated to be 'secure' against any single fault event that is regarded as credible by holding sufficient frequency response to avoid any interruption to electricity supply [10].

The European Network of Transmission System Operators for Electricity (ENTSO-E) classifies frequency management products as: frequency containment, restoration, and replacement reserves, each being called upon one after the other following a disturbance [11]. In GB at the time of writing, the frequency response services include primary, secondary, enhanced and high frequency responses. Primary frequency response is equivalent to ENTSO-E's frequency containment reserve, and secondary frequency response is equivalent to ENTSO-E's frequency restoration reserve, while high and enhanced frequency response services operate across both containment and restoration timescales. With the exception of enhanced frequency response (EFR), these services can be dynamic or static. Dynamic frequency responses are response services that continuously track frequency deviations and provide the required active power response, while static frequency responses are frequency-triggered services that discretely respond to frequency deviations. Figure 1 illustrates the definitions of GB frequency response services and demonstrates how the services contribute to containing a credible loss of infeed event. Detailed definitions of the services are available in [4] and [12].

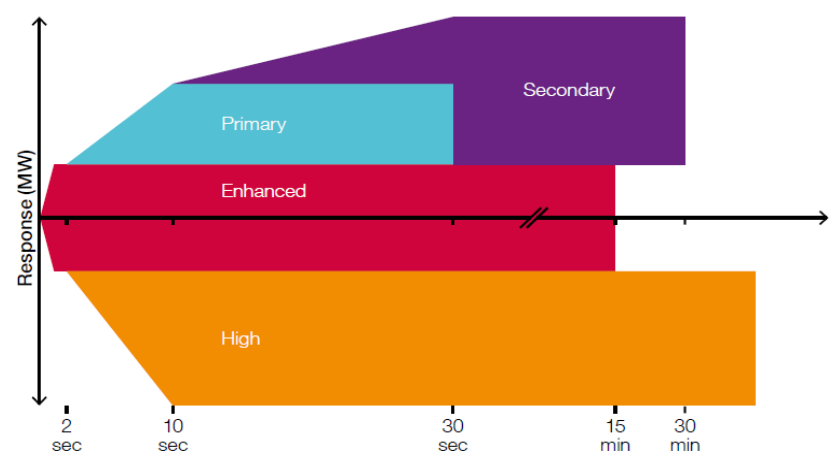

Figure 1: Current GB frequency response services [4].

In $\mathrm{GB}$, the electricity system operator (ESO) is required to set the operating state of the system such that particular sizes of single loss of infeed events do not lead to variations of system frequency outside certain limits detailed in the Security and Quality of Supply Standard (SQSS). These limits are defined by the loss risk classifications and a definition of unacceptable frequency conditions in [10]. Following a power imbalance, the GB ESO must also manage the RoCoF within limits defined by the Engineering Recommendation G59 [13], in order to prevent the undesirable tripping of loss of mains protection. It should be noted that, while the practical RoCoF limit in a future GB power system is likely to be $0.5 \mathrm{~Hz} / \mathrm{s}$, during operational scenarios that dispatch power plants with those RoCoF settings, or $1 \mathrm{~Hz} / \mathrm{s}$ at other times, at present there remains about $2 \mathrm{GW}$ of distributed generation using protection settings that could activate if RoCoF exceeds $\pm 0.125 \mathrm{~Hz} / \mathrm{s}$ [14]. 


\section{A FREQUENCY STABILITY MODEL}

Identifying the penetration limits of non-synchronous power dispatch requires the use of detailed engineering models to assess frequency behaviour over a large number of scenarios, while also considering and representing the range of factors that influence frequency. Many of these models are highly complex and make wide-ranging exploration of particular issues extremely challenging [15]. Thus, it is a well-established practice within the sector to use a variety of simplified models to address particular phenomena, provided they have been appropriately validated in respect of those phenomena. Here, a 'single bus' model, has been developed and setup to represent the frequency response of the GB power system, expanding on the principles outlined in [5, 16 - 19]. The 'single bus' model neglects the spatial distribution of generators and loads, and treats them as being connected to a single busbar. It is an aggregation of elements in the power system based on how they respond to frequency events, allowing for convenient representation of operational conditions and response providers whilst maintaining an accurate assessment of system frequency behaviour during a loss event. The single bus model, illustrated in Figure 2, is built on a platform provided by DigSILENT PowerFactory [20].

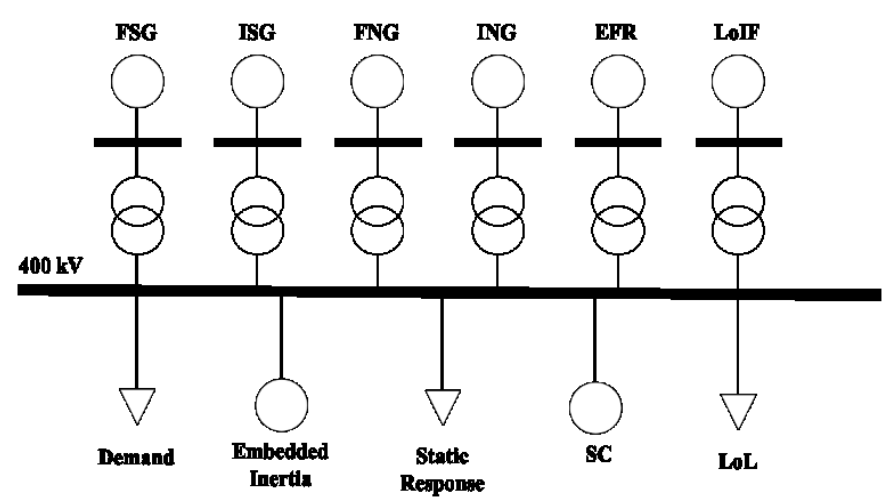

Figure 2: Single bus model.

\section{A. Key Elements of the model}

Figure 2 presents the key elements of the model. The FSG (Flexible Synchronous Generator) and FNG (Flexible Nonsynchronous Generator) elements of the model are the generation elements that provide active power response to a frequency imbalance via controller actions. As a synchronous machine, FSG also provides an inertial response to the frequency event, while FNG does not. The ISG (Inflexible Synchronous Generators) and ING (Inflexible Nonsynchronous Generators) elements of the model are generation elements with no controller action in response to a frequency event, however, ISG does provide an inertial response. It should be noted that FNG and ING can also include interconnector imports when applicable to the scenario. Within the dispatch, an inertia constant of 6 seconds is assumed for all gas units and 4 seconds for all other synchronous generators; these values are chosen following discussions with industry experts.

The EFR and Static Response elements represent their corresponding frequency services, while SC allows representation of Synchronous Compensators. The Demand element refers to demand on the transmission system, i.e. the power exported to the distribution network, and includes pumped hydro, interconnector exports and net unmetered embedded generation. The default value for the sensitivity of demand to frequency is $2.5 \% / \mathrm{Hz}$ [21]. The Embedded Inertia element represents the inertia associated with synchronous machines (generators and motors) operating within the distribution network. Based on discussions with industry experts embedded inertia is assumed to be equivalent to an inertia constant of 1.83 seconds as applied to the total transmission system demand. A loss of infeed event is represented by the LoIF element and a loss of load event is represented by the LoL element.

\section{B. Model Validation}

The model has been used to replicate the power imbalance experienced in the GB on the 9th of August 2019 as detailed in [22]. The public report of the event provides unusually complete details of the magnitude and timing of the loss events, the system conditions during the event and the magnitude of the frequency response that was provided by the GB ESO. The initial phase of the 9th of August event is simulated by applying these known parameters to the model alongside the underlying assumptions outlined above. Although the default assumption for dynamic primary response are its statutory requirements as defined in section II, in replicating the event, the speed of delivery of dynamic primary response is tuned given knowledge from discussions with industry experts that the real world delivery of the service usually slightly outperforms the statutory requirements. All other responses are modelled in line with their statutory definitions. The results of the simulation are compared with real 1 second frequency data from the time of the event in Figure 3. It is found that the comparative frequency and $\mathrm{RoCoF}$ traces of the simulated event are in close agreement with the real system measurements which acts as a strong validation of the model's ability to accurately replicate system frequency.



Figure 3: Replicating the 9th of August 2019 event.

With the model tuned to reproduce frequency containment behaviour during a power imbalance that closely matches that seen in real system, the single bus model is subsequently used for the frequency management studies presented in section IV. 


\section{METRICS FOR PENETRATION LiMITS}

At present, the GB ESO sometimes has to constrain the largest loss risk to manage power imbalances within RoCoF and frequency limits [4]. It should be noted that other actions could be taken by the system operator, including any one or a combination of the following: curtailing non-synchronous power; increasing system inertia; or dispatching additional response services.

In Ireland, a region experiencing similar concerns, the SNSP briefly described in section I is applied as a tool to manage the power system. SNSP is defined in (1), where 'Total Demand' includes interconnector exports.

$$
\text { SNSP }=\frac{\text { Wind }+ \text { Imports }}{\text { Total Demand }}
$$

The SNSP limit is the SNSP ratio that if exceeded would lead to a breach of frequency and RoCoF limits, unless corrective actions are taken by the system operator. Since the penetration limit is dependent on $\mathrm{RoCoF}$ and frequency containment, the key factors influencing it are the frequency and RoCoF limits, the amount and speed of energy responses in the power system (including inertia), and the size and type of the loss that is to be secured.

The following subsections consider the penetration limits derived for GB using the SNSP approach as defined in (1), alongside two alternative approaches. The model described in section III is used to conduct the studies needed to define the metrics. It should be noted that although the studies and results focus on $\mathrm{GB}$, the methodology used to produce these metrics is applicable to any power system concerned with frequency management limits.

Unless otherwise stated, the subsequent studies are conducted for operational scenarios in 2025 defined in Table I, using the tuned model from section III and the following additional assumptions:

- the loss of infeed is simulated as an instantaneous loss of power supply such that frequency is contained within $\pm 0.5 \mathrm{~Hz}$ of nominal frequency based on the normal loss frequency conditions as detailed in [8]. A loss risk of $1320 \mathrm{MW}$ is chosen for the normal loss event as this is the frequency condition for a future GB power system $[10,23]$;

- demand is modelled as total demand in the power system including exports;

- dynamic response services are simulated as defined, with 227 MW of EFR dispatched;

- static primary and secondary responses are both assumed to be dispatched at $250 \mathrm{MW}$ each;

- $\quad$ it is assumed that primary response is delivered by gas plants in the FSG element of the model, and frequency is contained using the least response reserve holding;
- the flexible synchronous generator is modelled as $70 \%$ loaded with $30 \%$ headroom for delivery of response;

- no response from flexible non-synchronous generation is assumed;

- generation background is based on the GB ESO's Two Degrees future energy scenario in [24]; and

- average availability of nuclear plants is assumed to be $77 \%$ for older plants and $95 \%$ for the newer plants [25].

TABLE I. Three Scenarios BASEd on Three RoCoF Settings

\begin{tabular}{|c|c|c|c|}
\hline Scenario & $\boldsymbol{A 1}$ & $\boldsymbol{A} \boldsymbol{2}$ & $\boldsymbol{A 3}$ \\
\hline Loss of Infeed (MW) & 1320 & 1320 & 1320 \\
\hline RoCoF Limit (Hz/s) & 1 & 0.5 & 0.125 \\
\hline
\end{tabular}

In constructing the operational scenarios, non-synchronous generation is dispatched first in the merit order, followed by flexible synchronous generation to meet the demand for primary response and securing the power system against the loss risk. Nuclear power is dispatched next, and any shortfall of power supply is met by dispatching the remaining synchronous generation.

\section{A. System Non-Synchronous Penetration Limit}

The SNSP metric, defined by (1), is applied to determine the penetration limits in $\mathrm{GB}$, for the three scenarios presented in Table I. For each demand level, the amount of nonsynchronous dispatch is increased until the containment limits for the event, in reference to frequency conditions and RoCoF limits, are breached. The value of non-synchronous dispatch achieved before the containment limits are exceeded defines the maximum amount of non-synchronous dispatch that the scenario can accommodate.

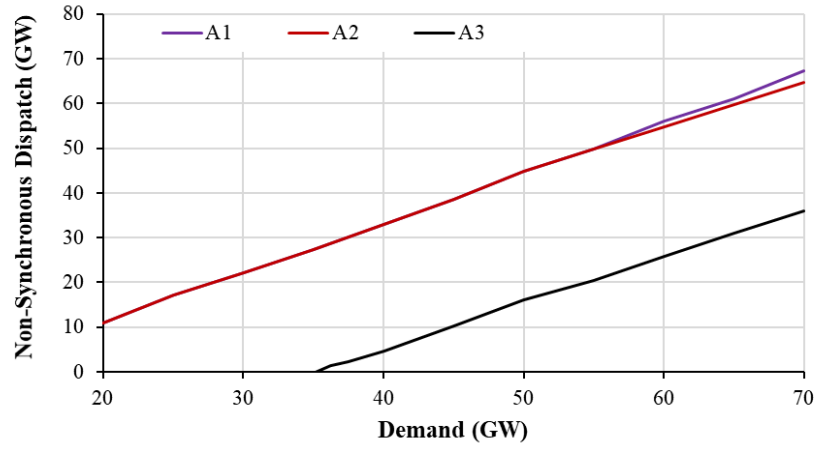

Figure 4: Penetration limits based on non-synchronous dispatch.

The results are presented in Figure 4, showing the trends of maximum non-synchronous power dispatch for a given demand level. The trend line produced for each scenario gives an expression for the SNSP limit in each scenario. It can be seen that at a higher RoCoF limit, for the same loss risk, the system can accommodate higher penetrations of non-synchronous dispatch at the same demand level when compared to the lower RoCoF limit. This is particularly true when comparing scenarios A1 $(1 \mathrm{~Hz} / \mathrm{s})$ or A2 $(0.5 \mathrm{~Hz} / \mathrm{s})$, for which there is little 
distinction between the calculated penetration limits, with A3 $(0.125 \mathrm{~Hz} / \mathrm{s})$. This is because as the RoCoF limit increases from $0.125 \mathrm{~Hz} / \mathrm{s}$ towards $0.5 \mathrm{~Hz} / \mathrm{s}$ or $1 \mathrm{~Hz} / \mathrm{s}$, the dominance of the RoCoF limit as the key containment factor reduces in favour of managing frequency within acceptable limits.

That said, when comparing A1 $(1 \mathrm{~Hz} / \mathrm{s})$ and A2 $(0.5 \mathrm{~Hz} / \mathrm{s})$ it can also be seen that at higher demand the SNSP in A1 is slightly higher than what is observed in A2. This is because at higher demand there is more contribution to frequency containment via the demand sensitivity characteristic. This factor, coupled with the higher RoCoF limit, permits A1 to accommodate slightly more non-synchronous dispatch in comparison to $\mathrm{A} 2$ at higher demand. The results for scenario A3, showing very low penetration limits, highlight the necessity for the removal of the existing RoCoF limit of $0.125 \mathrm{~Hz} / \mathrm{s}$ under future operating conditions. Failure to do so would imply very large re-dispatch costs. It should be noted that plans are in place to phase out the Loss of Mains protection assets by 2022, some of which are RoCoF based and impose a RoCoF limit on the system [26].

Although a useful metric for identifying the maximum nonsynchronous penetration in respect to containment limits, this representation of penetration limits is flawed. In particular, containment limits in terms of amount of non-synchronous power dispatched, is limited to the specific assumptions associated with the operational dispatch of the case being considered, i.e. the amount and speed of dispatchable (e.g. primary response) and inherent (e.g. inertia) energy responses assumed in the scenarios being considered. For instance, applying the limit of $4.75 \mathrm{GW}$ of non-synchronous dispatch at a demand of $40 \mathrm{GW}$ from scenario A3 ( 12\% SNSP), could result in an overestimation of containment capability if the same operational dispatch resulted in an inertia value less than what was used when defining the SNSP.

\section{B. Critical Inertia}

The limitation highlighted in section IV.A can be partially remedied by representing penetration limits in terms of critical inertia. In this case the inertia in the power system is progressively reduced until the lowest inertia required to contain the event is identified for a given demand level. This process is then repeated across a range of demand values, with the modelling assumptions unchanged from the previous study.

The results presented in Figure 6, show inertia against demand instead of the amount of non-synchronous power dispatched against demand, where inertia in Figure 6 is the critical inertia required to contain the loss event, given the other energy responses that are available at the time of the event. The critical inertia is ratio, GVAs/GW, described by (2), where the plots in Figure 6 can be described as expressions defining the critical inertia limits for each scenario. This method produces a metric that defines the penetration limits by identifying the critical inertia for a given demand beyond which frequency and RoCoF conditions are breached during a loss event, i.e. the containment limit.

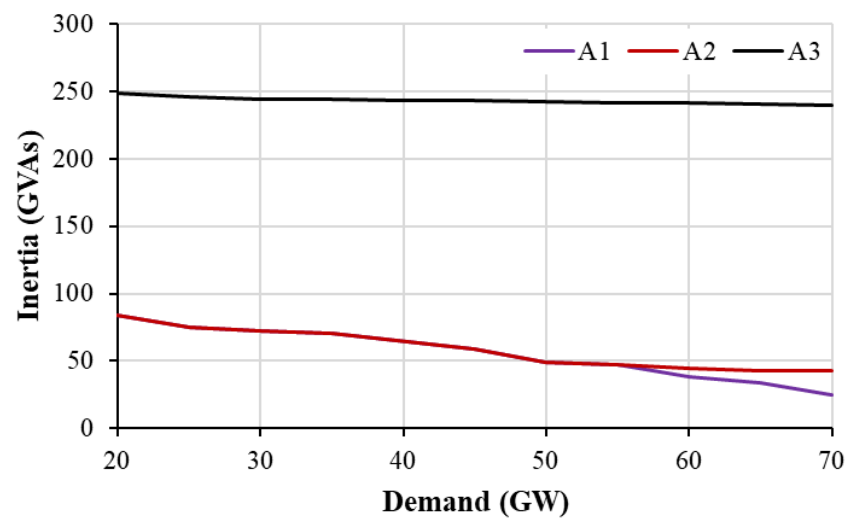

Figure 5: Penetration limits based on inertia.

$$
\text { Critical Inertia }=\frac{\text { Inertia }}{\text { Total Demand }}
$$

Upon considering scenarios A1, A2 and A3, a similar behaviour as observed in Figure 4 is seen in Figure 6, particularly in reference to the comparative trends of the scenarios. As with the Figure 4, in Figure 6, there is a marked distinction between the critical inertia trend line observed in A3 $(0.125 \mathrm{~Hz} / \mathrm{s})$ in comparison to A1 and A2. This highlights the previous assertion that the $\mathrm{RoCoF}$ limit is the dominant containment component in A3, with the dominance shifting towards frequency containment in A1 and A2. The slight difference at higher demand observed when comparing A1 and $\mathrm{A} 2$ is due to the higher RoCoF limit in A1 and the contribution to containment from demand sensitivity.

Although this representation of containment limits improves on the failings of the previous, in respect to variations in inertia across similar penetration limits, it also has its flaws. It only addresses variations in inertia, making no improvement on variations in demand sensitivity and dispatchable energy responses.

\section{Containment Component Metric}

Defining the containment limits of an operational dispatch by considering the containment components addresses the limitations previously described, i.e. by separately considering penetration limits in terms of the energy responses that dominate RoCoF containment and those that dominate frequency containment. In this manner, RoCoF and frequency limits are treated as individual components of containment limit, as discussed in the rest of this section

Identifying whether a scenario is likely to exceed RoCoF limits, can be done using (3) below, where the instantaneous RoCoF, $g$, at the inception of the event is a function of the power imbalance $\Delta P$, at nominal frequency $f_{0}$, where $H_{t}$ is the total inertia of the system for a given operational dispatch. 


$$
g=\left(\frac{\Delta P \times f_{0}}{2 \times H_{t}}\right)
$$

The total inertia of a system depends on the specific generation dispatch and the embedded inertia. Equation (4) captures the total inertia of the system using inertial contributions from inflexible generation (e.g. nuclear plants), flexible generation (e.g. gas plants) and embedded inertia (as defined in section III.A).

$$
H_{t}=\mathrm{FSG}_{M V A S}+I S G_{M V A S}+\text { Embedded }_{M V A s}
$$

The three components in the RHS of (4) are defined as follows:

$$
\begin{gathered}
S G_{\text {supply }}=T_{g}-T_{n} \\
F S G_{M V A s}=\left(\frac{S G_{\text {supply }} \times F G_{\text {perc }}}{L_{1} \times p f_{1}}\right) \times H_{F S G} \\
I S G_{M V A s}=\left(\frac{S G_{\text {supply }} \times I G_{\text {perc }}}{L_{2} \times p f_{2}}\right) \times H_{I S G} \\
\text { Embedded }_{M V A s}=\text { Demand } \times H_{\text {embedded }}
\end{gathered}
$$

Equation (5) works out the amount of the dispatched synchronous generation $\left(S G_{\text {supply }}\right)$ from the difference between total generation dispatch $\left(T_{g}\right)$ and total non-synchronous dispatch $\left(T_{\mathrm{n}}\right)$. The inertia in MVAs of the flexible and inflexible synchronous generation elements are represented by $F S G_{M V A S}$ and $I S G_{M V A S}$ respectively, and defined in (6) and (7), where the percentage loading of the units $\left(L_{1}\right.$ and $\left.L_{2}\right)$ defines the rating of the units based on the power factors $\left(p f_{1}\right.$ and $\left.p f_{2}\right)$. The corresponding inertia constants $H_{F S G}$ and $H_{I S G}$ account for the mixture of the different inertia constants by fuel type, for a percentage of the dispatched synchronous generation that is flexible $\left(F G_{\text {perc }}\right)$ or inflexible $\left(I G_{\text {perc }}\right)$. Equation $(8)$ defines the embedded inertia in MVAs as the product of the demand in MW and the embedded inertia constant $\left(H_{\text {embedded }}\right)$ in seconds.

In order to provide an accompanying containment relationship for the frequency component, a range of scenarios are considered to produce a trend in terms of active power response and instantaneous RoCoF, denoted as $\mathrm{g}$. To produce results in this format, a second set of simulation studies are conducted. It is assumed here that all frequency response is delivered by the enhanced frequency response service, and, with the exception of inertia, no other energy response is available to contain the event. In particular, the contribution from demand sensitivity is not considered at present but will be accounted for in future work.

A constant demand level of $30 \mathrm{GW}$ is chosen, while containment limits are assessed for a range of inertia values. It should be noted that a range of demand is not needed for this study, since demand sensitivity is not presently being considered and frequency response is only delivered by EFR. It should also be noted that, for the purposes of this study, in instances where additional inertia is required for a given instantaneous RoCoF operational scenario, additional inertia is provided by synchronous compensation. The resultant trends are extrapolated for a range of loss risk values, based on the loss risk frequency conditions in [10], producing a surface function that determines the EFR reserve that needs to be held to keep frequency within limits for a given loss risk and instantaneous RoCoF. The equations of the surfaces were determined using a least regret fit; meaning that the curve of both surfaces sit above the data points in the reserve axis. Therefore, the reserve determined for combinations of instantaneous RoCoF and loss risk values will be greater than or equal to the amount of reserve determined in the individual simulation study. This was done because a best fit without least regret gives some combinations of RoCoF and loss risk that would result in a prediction of reserve less than what would be observed in the simulation. The resultant surfaces are shown in Figure 6, with associated expressions shown in (9) and (10), and the values of the constants are shown in Table V. Equations (9) and (10) are expressions for the infrequent and normal loss risk conditions respectively. These two expressions represent the penetration limits for both loss risk frequency conditions, where $\mathrm{g}$ is the instantaneous RoCoF, $\Delta P$ is the loss risk, $r_{e}$ is the EFR, and $g_{t h}$ is the threshold for the applicable constants. Where $g \leq g_{t h}$ defines the lower bound and $g>g_{t h}$ defines the upper bound. The upper and lower bounds split each loss risk condition, as depicted in Figure 6, into two expressions that describe both parts of the whole surface.

Equations (3), (9) and (10) can be used together to first constrain the power system within the RoCoF constraint via (3) and then based on the resultant $\mathrm{g}$, the frequency constraint can be determined using either (9) or (10), depending on the loss risk condition. This metric, expressed as a set of equations, can be used to determine the minimum amount of reserve that would need to be held, if EFR was the only energy response available to contain a given loss risk for an operational scenario at a given system inertia, represented here by instantaneous RoCoF. Similarly, the metric can also be used to determine how much inertia needs to be available for a given amount of enhanced response and loss risk value, or the maximum loss risk value for a given amount of inertia and EFR. Considering the limits to penetration in this manner, shows that the amount of non-synchronous power dispatched is not an inherent limitation to containment and frequency management. Instead, the factors most dominant are the energy responses available, i.e. dispatchable services such as EFR and inherent responses such as inertia, and the size of the loss risk. 

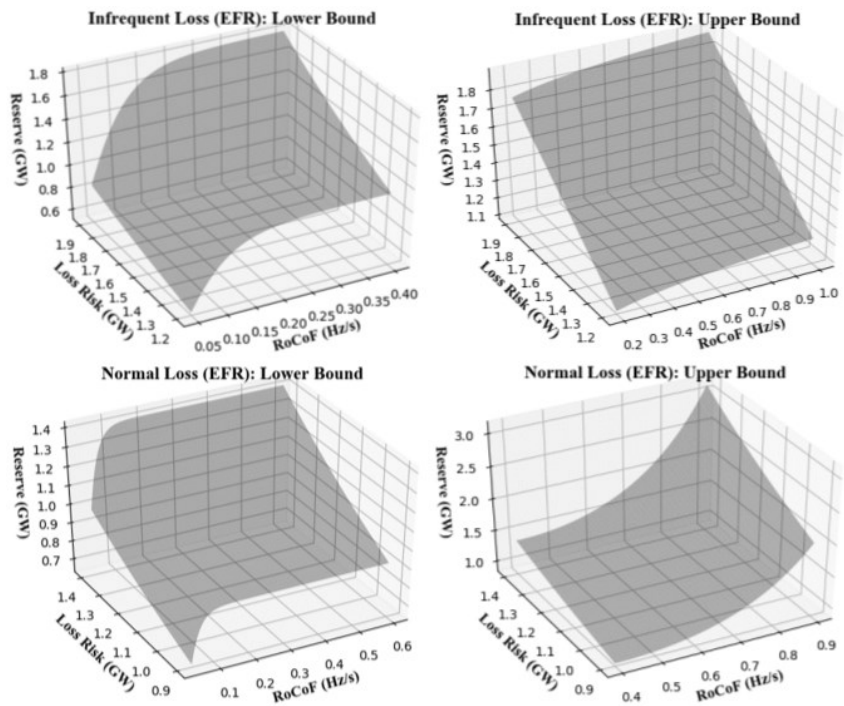

Figure 6: Surface plots for Enhanced response showing infrequent loss risks and normal loss risks both below (Lower Bound) and above (Upper Bound) the RoCoF threshold.

$$
\begin{gathered}
a_{5}^{e}= \begin{cases}a_{1}^{e} e^{a_{2}^{e} g}+a_{3}^{e} \Delta P+a_{4}^{e} \ln r_{e}, & g \leq g_{t h} \\
a_{1}^{e} \ln g+a_{2}^{e} \Delta P+a_{3}^{e} e_{4}^{a_{4}^{e} r_{e},} & g>g_{t h}\end{cases} \\
a_{5}^{e}=\left\{\begin{array}{rr}
a_{1}^{e} e^{a_{2}^{e} g}+a_{3}^{e} \Delta P+a_{4}^{e} \ln r_{e}, & g \leq g_{t h} \\
a_{1}^{e} g^{2}-a_{2}^{e} g+a_{3}^{e} \Delta P+a_{4}^{e} \ln r_{e}, & g>g_{t h}
\end{array}\right.
\end{gathered}
$$

TABLE II. CONSTANTS FOR SURFACE EQUATIONS

\begin{tabular}{|c|c|c|}
\hline \multicolumn{3}{|c|}{ Infrequent loss risk } \\
\hline Constant & Lower Bound & Upper Bound \\
\hline$g_{\text {th }}$ & \multicolumn{2}{|c|}{0.3000} \\
\hline$a_{1}^{e}$ & $1.4420 \times 10^{0}$ & $6.5414 \times 10^{-3}$ \\
\hline$a_{2}^{e}$ & $-2.1523 \times 10^{1}$ & $8.2146 \times 10^{-2}$ \\
\hline$a_{3}^{e}$ & $-4.2483 \times 10^{-1}$ & $-1.5089 \times 10^{0}$ \\
\hline$a_{4}^{e}$ & $6.4720 \times 10^{-1}$ & $5.1913 \times 10^{-2}$ \\
\hline$a_{5}^{e}$ & $-4.2336 \times 10^{-1}$ & $-1.5091 \times 10^{0}$ \\
\hline \multicolumn{3}{|c|}{ Normal loss risk } \\
\hline Constant & Lower Bound & Upper Bound \\
\hline$g_{\text {th }}$ & $4.1135 \times 10^{0}$ & $-1.0878 \times 10^{1}$ \\
\hline$a_{1}^{e}$ & $-4.6323 \times 10^{1}$ & $-8.8867 \times 10^{0}$ \\
\hline$a_{2}^{e}$ & $-1.5332 \times 10^{0}$ & $-2.8890 \times 10^{0}$ \\
\hline$a_{3}^{e}$ & $1.7888 \times 10^{0}$ & $3.2090 \times 10^{0}$ \\
\hline$a_{4}^{e}$ & $-1.5267 \times 10^{0}$ & $-1.1907 \times 10^{0}$ \\
\hline$a_{5}^{e}$ &
\end{tabular}

It should be noted that in power systems, the RoCoF observed by relays, such as Loss of Mains protection, differs from the instantaneous RoCoF value calculated using (3), which means that constraints using this method would be conservative in their assessment of the RoCoF component of the containment limit. In addition, the results presented in this paper consider EFR to be the only available dispatchable energy response service at the time of the event. This means that other energy responses would need to be equated to EFR, to determine whether or not the energy responses available, including those from other dispatchable services, e.g. primary response, would adequately contain the event. It should also be noted that in generating (9) and (10), modifications such as the inclusion of demand sensitivity or different frequency conditions would change the value of the constants presented in Table II, and a system operator applying this metric would need to first generate the expressions before they can be used for system management and planning. However, once the expressions have been generated, they can be applied to a wide range of operational scenarios without requiring further simulations unlike the other methods previously described.

\section{CONCLUSIONS}

A simplified frequency stability model is presented, and it is validated against the loss event in GB on August 9th, 2019. The results of the validation demonstrate the capability of the model to accurately capture the frequency behaviour of the power system. The need to understand the relationship between the penetration of non-synchronous power dispatch and containment limits are discussed, and the limitations of using the current SNSP metric is presented.

The following are the key conclusions surrounding the subject of this paper:

1. While a useful metric, a flaw has been identified in representing containment limits in terms of the amount of non-synchronous power dispatched. Specifically, the SNSP metric neglects the variation in available energy response for a given operational dispatch when comparing two otherwise identical SNSP system conditions. For instance, those two conditions could have very different amounts of inherent (e.g. inertia) and dispatchable (e.g. EFR) energy responses, depending on the operational dispatch.

2. This flaw is addressed in part by using a representing of containment limits in terms of inertia. This improvement, however, does not account for variation in other forms of energy response that can change depending on the dispatch of the operational scenario.

3. Separating containment limits into components of RoCoF and acceptable frequency conditions offers further improvement on the previous methods. Although the methodology will need to be followed to generate expressions before being applied to a given power system, the component method offers the potential for more flexibility in terms of understanding and quantifying the factors that contribute to the containment limits.

4. The variations in inherent and dispatchable energy responses, alongside the size of the loss risk and the applied frequency and RoCoF limits are the dominant factors in defining containment limits, such that a 
change to any of these factors influence containment and frequency behaviour. The amount of nonsynchronous power dispatched is not an inherent limitation to containment and frequency management.

Further work is required to develop the containment components metric, and to compare the performance of all three metrics. In particular, the contribution of demand sensitivity to containment components metric will be included in future work. A means of equating energy responses will also be considered, allowing the expressions generated to be used in a tool that can consider the combination of energy responses available at the time of the event. Future work will also include expanding the SNSP and critical inertia into a 3-dimensional surface that can be used to determine the redispatch costs when these metrics are applied as a constraint on the dispatch of the power system.

\section{REFERENCES}

[1] European Commission, "Towards a sustainable and integrated Europe: Report of the Commission Expert Group on electricity interconnection targets," 11 2017. [Online]. Available: https://tinyurl.com/y5k2zt5m. [Accessed 2709 2019].

[2] M. Joos and I. Staffell, "Short-term intergration costs of variable renewable energy: Wind curtailment and balancing in Britain and Germany," Renewable and Sustainable Energy Reviews, vol. 86, pp. 45 $-65,2018$.

[3] EirGrid and SONI, "Annual Renewable Energy Constraint and Curtailment Report 2018," 05 2019. [Online]. Available: https://tinyurl.com/y5325gy5. [Accessed 0410 2019].

[4] National Grid ESO, "System Operability Framework November 2016," 11 2016. [Online]. Available: https://tinyurl.com/y3fadel5. [Accessed $26062019]$.

[5] J. O'Sullivan, A. Rogers, D. Flynn, P. Smith, A. Mullane and M. O'Malley, "Studying the Maximum Instantaneous Non-Synchronous Generation in an Island System-Frequency Stability Challenges in Ireland," IEEE Transactions on Power Systems, vol. 29, no. 6, pp. 2943 - 2951, 2014.

[6] EIRGRID and SONI, "Operational Constraints Update 30/04/2018," 4 2018. [Online]. Available: https://tinyurl.com/y3flv3b5. [Accessed 26 06 2019].

[7] M. Nedd, Q. Hong, K. Bell, C. Booth and P. Mohapatra, "Application of synchronous compensators in the GB transmission network to address protection challenges from increasing renewable generation," in 2017 CIGRE B5 Colloquium, Auckland, New Zealand, 2017.

[8] National Grid ESO, "Zero carbon operation of Great Britain's electricity system by 2025," 0104 2019. [Online]. Available: http://tinyurl.com/y56rnxg4. [Accessed 2806 2019].

[9] CIGRE Working Group C1.17, "Planning to manage power interruption events," in Technical Brochure 433, Paris, CIGRE, October 2010.
[10] National Grid, "National Electricity Transmission System Security and Quality of Supply Standard," 802 2017. [Online]. Available: https://tinyurl.com/y43vdub5. [Accessed 1802 2019].

[11] Enstoe, "Network Code on Load-Frequency Control and Reserves," 28 June 2013. [Online]. Available: https://tinyurl.com $/ \mathrm{m} 2 \mathrm{hkmeu}$. [Accessed 1905 2017].

[12] National Grid, "Enhanced Frequency Response Seminar," 02062016. [Online]. Available: https://tinyurl.com/hseso8w. [Accessed 0102 2017].

[13] Energy Networks Association, "Engineering Recommendation G59 Issue 3 Amendment 6," 1606 2019. [Online]. Available: https://tinyurl.com/y5xf32ok. [Accessed 2608 2019].

[14] Ofgem, "Appendices to the Technical Report on the events of 9 August 2019," 0609 2019. [Online]. Available: https://tinyurl.com/y3oahr5f. [Accessed 2609 2019].

[15] K. R. W. Bell and A. N. D. Tleis, "Test system requirements for modelling future power systems," in General Meeting of the Power and Energy Society (PES), Minneapolis, Minnesota, 2010.

[16] National Grid, "GC022 - Frequency Response," 0901 2013. [Online]. Available: https://tinyurl.com/y65gmkaz. [Accessed 2109 2017].

[17] M. Yu, A. J. Roscoe, A. Dyśko, C. D. Booth, R. Ierna, J. Zhu and H. Urdal, "Instantaneous penetration level limits of nonsynchronous devices in the British power system," IET Renewable Power Generation, vol. 11, no. 8, pp. 1211 - 1217, 2016.

[18] K. Máslo, A. Kasembe, S. Moroni and R. Pestana, "Frequency Stability Modelling of the Future Continental Europe Power System," in 52nd International Universities' Power Engineering Conference, Heraklion, 2017.

[19] J. Undrill, "Primary frequency response and control of power system frequency," 02 2018. [Online]. Available: https://tinyurl.com/yboca8fh. [Accessed 2201 2019].

[20] DigSilent, "PowerFactory," 2017. [Online]. Available: https://tinyurl.com/nxe4fta. [Accessed 1905 2017].

[21] National Grid, "System Operability Framework 2016," 2016. [Online]. Available: https://tinyurl.com/yad6pju8. [Accessed 1809 2017].

[22] Ofgem, "Investigation into 9 August 2019 power outage," 082019. [Online]. Available: https://tinyurl.com/y2ndkayw. [Accessed 1909 2019].

[23] Ofgem, "Letter: National Electricity Transmission System Security and Quality of Supply Standard: Normal Infeed Loss Risk (GSR015)," 2014. [Online]. Available: https://tinyurl.com/ybd969d4. [Accessed 18 $012018]$.

[24] National Grid ESO, "Future Energy Scenarios (2019)," 072019. [Online]. Available: https://tinyurl.com/y6d67ouf. [Accessed 1909 2019].

[25] Department for Business, Energy and Industrial Strategy, "Nuclear capacity in the UK," 03 2017. [Online]. Available: http://tinyurl.com/yyody2h7. [Accessed 1302 2019].

[26] Energy Networks Association, "Accelerated Loss of Mains Change Programme (ALoMCP)," 2019. [Online]. Available: https://tinyurl.com/y5pr6n4b. [Accessed 0310 2019]. 